\title{
The secretory senescence of the airway
}

\author{
Alessandro Maselli del Giudice ${ }^{1}$, Matteo Gelardi², Pier Gerardo Marano², \\ Aurelio D’Ecclesia ${ }^{3}$, Giuseppe Campobasso ${ }^{1}$, Francesco Cariti', \\ Antonio Palumbo ${ }^{3}$, Michele Loglisci4 ${ }^{4}$ Marco de Vincentiis ${ }^{5}$, \\ Michele Cassano ${ }^{2}$, Francesco Barbara ${ }^{1}$ \\ ${ }^{1}$ Department of Otorhinolaryngology, Mons. Di Miccoli Hospital, Barletta, Italy; \\ ${ }^{2}$ Department of Otorhinolaryngology, University of Foggia, Italy; ${ }^{3}$ Department of Otorhinolaryngology, \\ "Casa Sollievo della Sofferenza" Hospital, San Giovanni Rotondo, Italy; \\ ${ }^{4}$ Department of Otorhinolaryngology "Vito Fazzi", Lecce, Italy; \\ ${ }^{5}$ Department of Otorhinolaryngology “Umberto I" Hospital, Rome, Italy
}

Received: March 21, 2020

Accepted: June 3, 2020

\section{Correspondence}

Alessandro Maselli del Giudice

Ospedale Mons Dimmicoli

via Ippocrate 15,76121 Barletta, Italy

E-mail: ale.maselli@tiscali.it

\section{Conflict of interest}

The Authors declare no conflict of interest

How to cite this article: Maselli del Giudice A, Gelardi M, Marano PG, et al. The secretory senescence of the airway. Journal of Gerontology and Geriatrics 2020;68:61-68. https://doi. org/10.36150/2499-6564-482

C Copyright by Società Italiana di Gerontologia e Geriatria (SIGG)

\section{(c) (i) (3) $\odot$}

\section{OPEN ACCESS}

This is an open access article distributed in accordance with the CC-BY-NC-ND (Creative Commons Attribution-NonCommercial-NoDerivatives 4.0 International) license. The article can be used by giving appropriate credit and mentioning the license, but only for non-commercial purposes and only in the original version. For further information: https://creativecommons.org/licenses/by-nc-nd/4.0/deed.en
Secretory senescence of the upper and lower airway represents the most important risk factor for respiratory complication in the elderly. In most cases, patients present with high health care costs due to the relevant number of hospitalizations and medical cares.

A growing evidence is accumulating on the role of immunosenescence in the pathogenesis of chronic rhinosinusal disorders, and on defect in the lubrication of the corneal, conjunctival and scleral mucosa, delicate structures of the external eye, and of trachea-bronchial diseases, dependent on a failure of the gland system and reduction of mucociliary clearance (MCC). The presence of multiple comorbidities in the elderly may significantly contribute to a systemic pro-inflammatory state which negatively affects the airway. Secretory senescence promotes systemic inflammation and exacerbates the pre-existing pathologies. The role of cytokines is associated to a pro-inflammatory state of breathing apparatus. Several observations support the hypothesis that the secretory senescence can act in a paracrine and vasocrine manner to influence the structure and function of glands, mucus and lacrimal secretions, thus contributing to fraility and hospitalizations in the elderly. Given the recognized role of secretory senescence in the pathophysiology of airway disease, it should be desirable to identify specific therapies targeting the nasal, lacrimal and tracheo-bronchial secretory system able to modulate its pro-inflammatory profile and the negative effect of the inflammatory burden on the elderly.

Key words: immunosenescence, mucociliary clearance, nasal mucus felting, lacrimal system, Meibomian glands, tracheo-bronchial tract

\section{INTRODUCTION}

Epidemiology has shown that the elderly is more susceptible to respiratory illness, and advanced age appears to predispose older individuals to the increased severity of respiratory infections. Chronic rhinosinusitis in old patients is linked to immune functional changes with age, the immunosenescence, depending on impaired cell-mediated immunity and secretory senescence. These factors are implicated in cancer, higher probability of complications like cardiovascular illness, respiratory complications and challenging management of physical and psychological conditions. The modifications of epithelium and mucus film is common in several districts 
of the upper airways in the elderly as the lacrimal system, and its consequence, the dry eye disease, is a very common disorder that affects the elderly population. This causes a defect in the lubrication of the corneal, conjunctival, and scleral mucosa. Aging represents a gradual deterioration of organization at the molecular, cellular, tissue, organ, and system level of the body. The increased incidence of respiratory diseases in the old age is due to cellular, molecular and structural alterations based on several mechanisms and includes alteration in immune response, clearance mechanisms (cough and mucociliary clearance), and inflammatory cytokine secretion. The importance of each of these risk factors vary between individuals. So, to reduce the incidence and severity of respiratory diseases in the elderly, it is necessary to understand the effect of aging on these systems. Even if biological aging is normally linked to chronological age, it can occur earlier in life, in a way partially independent from an individual's chronological age. Markers that are used to describe biological aging are also generally related to chronic inflammation. These alterations also involve the low airways and can be dangerous for chronic inflammation.

\section{THE RHINOSINUSAL DISTRICT}

The rhinosinusal district consists of a pseudostratified ciliated epithelium with mucipar goblet cells covered by a mucus film. Both the epithelium and the mucus film are involved in the senescence process with changes in the composition of the mucus and in the relationship between ciliated and mucipar goblet cells ${ }^{1}$.

Geriatric rhinitis is an inflammation of the nasal mucosa with multifactorial etiology, in which different alterations of cellular types and characteristic of mucus can be involved. This inflammation can be due to viruses, bacteria, irritants or allergens that produce large variation in several aspects of mucous, resulting in runny nose or postnasal drip. In the elderly, nasal mucosa atrophies because of the recession of the collagen fibres and loss of elastic fibres in the skin. Fibrosis and atrophies were correlated with the expression of HSP47 gene. The weakening or thinning of these connective tissues in the nose can cause the tip of the nose to droop which restricts airflow with subsequent nasal obstructions. Some of the symptoms of geriatric rhinitis in addition to a runny nose include loss of smell, crustiness in the nostrils and a dry cough or epistaxis. A runny nose is very common in individuals with Lewy body dementia and Parkinson's disease, and common in the later stages of Alzheimer's disease. Elderly persons present reduced nasal mucosal sensitivity with regard to trigeminal, chemosensory irritation and they could present a decreased sensitivity of intranasal trigeminal nerve endings due to trophoneurotic atrophy as possible reason for difficult nasal breathing. ${ }^{2}$ Furthermore, nasal complaints could be correlated to impaired intranasal air conditioning, due to decreased heat and water exchange. The age-related alterations therefore could lead to intranasal climatization alteration. The reduction of the water component of the mucus film would favour the action of irritating agents. Nasal mucus contains $90 \%$ water and glycoproteins, as well as ions (sodium 128-150 mmol, potassium 17-41 mmol, calcium $4 \mathrm{mmol}$, magnesium $5 \mathrm{mmol}$, chloride139 mmol). The right balance of all components of the nasal mucus will allow the formation of the phenomenon known as "Ferning" observable by a phase contrast microscope. It is produced by submucosal, seromucous glands, goblet cells, transudation of blood plasma, mucosal tissue fluid and tear fluid. Due to transudate, most proteins detectable in serum may also be showed in nasal secretions. In cases of local inflammation, the amount of transudate and their respective proteins will increase. The mucus layer will be moved at a velocity of $2-25 \mathrm{~mm} /$ min. In the elderly, the aqueous component decreases and the mucociliary transport time increases.

At microscopic level, the phenomenon of ferning will be reduced until not realising itself, as it happens in the different forms of rhinopathies ${ }^{4}$.

Mucociliary clearance (MCC) is the first line of defence in both the upper and lower airways. Efficient MCC, whereby debris and pathogens trapped in the mucus are cleared from the airways by ciliary beating, depends on the proper functioning of three major systems:

1 the synthesis and secretion of mucin and other mucus proteins;

2 the proper hydration of the airway surface, and

3 the coordinated activity of the cilia.

A perturbation in any of these components can result in impaired $\mathrm{MCC}^{3}$.

Patients with primary ciliary dyskinesia, caused by a mutation in genes necessary for the proper formation or function of cilia, suffer from recurrent/chronic rhinosinusitis, otitis media, and pulmonary infections and bronchiectasis disease. In cystic fibrosis, mutations in the CFTR gene cause a defect in $\mathrm{Cl}^{-}$and hence water secretion that results in dehydration of mucus and impaired MCC that leads to chronic pulmonary infections, bronchiectasis, and eventually lung failure. Runny nose can be caused by environmental irritants, such as pollution, dust, tobacco smoke, perfume, scented candles, or oils. Central heating can exacerbate the condition. If not an environmental factor, a runny nose could be caused by allergies to pets, a new detergent or fabric softener, a new soap or shampoo, or foods. Geriatric rhinitis is a familiar and bothersome condition in aging 
adults, can cause headaches and diminished concentration. Some individuals have chronic nose drips that have no underlying cause. This condition is called nonallergic rhinitis. Individuals typically sneeze a lot and experience nasal congestion, symptoms much like hay fever. However, the individual is not allergic to anything. Though little can be done about this condition, the caregiver can identify triggers that could possibly make the nasal drip worse, such as certain odours, medications, changes in diet or food, and the weather.

In the elderly, the nasal mucosa involves a reduced number of goblet cells and a reduced number of elastic fibres and structures may contribute to a more pronounced feeling of a dry nose, recurrent intranasal crusting, postnasal dripping, and obstructed nasal breathing. Data on intranasal climatization in elderly persons show temperature and humidity reduced. This reduction of humidity is accompanied by a reduction in the local vascularization of the turbinate mucosa, shown as well in the picture of atrophic rhinitis by rhinoendoscopy.

Aging significantly increases the vulnerability to rhinosinusal disorders, but there are few studies investigating the key factors in aging that affect the rhinosinusal tract. Most of these investigate the microbiota and host gene expression changes in association with ageing. In the elderly, at the level of the mucous membranes of the upper airways, there is a reduction of secretory $\lg A$, which is due to both age-related immunosenescence and to the reduction of local vascularization and chronic use of drugs as well evidenced by the studies about immunosenescence. Immunosenescence is defined as changes in the innate and adaptive immune response associated with increased age.

Additionally, the airway epithelial cells produce nitric oxide, secrete several cytokines, in particular type I and III IFNs, growth factors such as granulocyte/macrophage colony-stimulating factor, IgA and several antimicrobial proteins. There are notable changes in the airway epithelium, as well as for the lower airways, including the nose, in patients also with disruptions of the epithelial cell tight junctions, increased susceptibility to apoptotic effects of oxidants, and impaired production of IFN- $\beta$ and IFN- $\alpha$, which promote epithelial damage and increase the susceptibility to viral infections.

Nasal epithelial tissue collected from non-smoking healthy individuals (ages 11 to 90 years) without respiratory disease demonstrated a significantly decreased ciliary beat frequency and clearance with age accompanied by microtubular disarrangements. This was linked to increased apoptosis of goblet cells in the upper airway. The barrier function of nasal mucus was also compromised and the microbiota were frequently observed in contact with the epithelium. Lysozyme was expressed in significantly reduced amounts. These barrier defects were accompanied by major changes in the microbiota and were negatively affected by old age in human beings. Transcriptomics revealed age-associated decreases in the expression of immunity and other genes in nasal mucosal tissue, including decreased $T$ cell-specific transcripts and $T$ cell signalling pathways. The physiological and immunological changes we observed in old age could have major consequences beyond the gut.

Very little information is available regarding Nasal $\mathrm{Mu}$ cociliary Clearance (NMC) and the effect of age on this defence mechanism. For this reason, a study P. Paul e P. Johnson was conducted to assess the effect of age on nasal mucociliary clearance in a population of rural women. This study showed that NMC time increases with increasing age and that defences by mucociliary clearance mechanisms are impaired with age. Impairment of NMC with age was found to be higher among lower socioeconomic status, lower literacy status, and biomass fuel users. Anatomical changes that occur with ageing include nasal mucosal damage which has accumulated from infections over the years, ciliary ultrastructural defects such as the occurrence of increased central microtubular disorientation, age-related changes resulting in altered proportions of elastic tissues and collagen, and alterations in the cross-linkage of ageing collagen. Mucociliary clearance became abnormally slow or uncoordinated ciliary beating, where neighbouring cilia do not beat in a coordinated fashion and in the same direction. Biochemical changes such as greater oxidative stress potential may play a role as well. Chronic rhinosinusitis in old patients is linked to immune function changes with age, immunosenescence, depending on impaired cell-mediated immunity due to thymus involution. A lot of pathogenic mechanisms are proposed for including oxidative stress and elevated reactive oxygen species, especially in old age. These factors are implicated in cancer, higher probability of complications, like cardiovascular illness, respiratory complications, and challenging management of physical and psychological conditions. High concentrations of uric acid in the upper airways are achieved through active secretion of uric acid from cells lining the respiratory tract. There is a two-layer structure, comprising a lower aqueous Sol phase and an upper mucus, Gel phase. Peden et al. have demonstrated that Sol phase uric acid concentration is increased in the nasal cavity following cholinergic stimulation of the airways. Furthermore, they demonstrated that the increase in uric acid correlated positively with lactoferrin low concentration. As lactoferrin is predominately derived from mucosal gland secretions (Gel phase) this supports the contention that uric acid in the upper airways is derived, in part, 
from glandular secretions. Uric acid secretion in this gel phase may therefore be closely related to mucus secretion. Cellular secretion is probably involved, coupled with poor re-absorption mechanisms. If antioxidant defences are indeed compromised in older people, even in the absence of airways disease, this is likely to increase their sensitivity to air pollution. Studies of the airways in asymptomatic elderly humans suggest that inflammatory cell numbers are increased in comparison with younger volunteers. This low-grade inflammation may influence antioxidant concentrations through increased free radical fluxes in the airways. Ageing has also been associated with increased interleukin-6 concentrations and superoxide production in older subjects. These findings have been interpreted to indicate that low-grade inflammation does indeed exist in the respiratory tract of many asymptomatic elderly ${ }^{5}$. The reduction of blood flow to the nasal mucosa contributes to local atrophy and leads to an expansion of the nasal volume with loss of the ability to humidify the inspired air and the appearance of a paradoxical nasal congestion sensation. Atrophic rhinitis can overlap other forms of rhinitis, both allergic and non-allergic, or may represent their evolution. As regards the various pictures of nasal cytology, allergic rhinitis is characterized by an inflammatory infiltration of neutrophils, mononuclear cells (lymphocytes and monocytes), eosinophils and partly mast cells degranulated, without differences between the elderly and younger patients ${ }^{6}$. In elderly patients with persistent rhinitis, the cytologic aspect is characterized by numerous neutrophils and rare eosinophils, which could suggest the presence of minimal persistent inflammation in the nasal mucosa. Note that in the study reported by the work of prof. Gelardi, the ratio between ciliary cells and goblet cells was significantly lower than in young adults. The relative reduction in the ratio of ciliary cells / goblet cells in favour of goblet cells is associated with an increase in the presence of bacteria and the formation of biofilms (Figs. 1-2) which is probably the consequence of a slower clearance of the ciliary mucosa. The cellular alterations of the epithelium and the variations of the secretory component of mucus lead to a higher incidence of infections in the elderly, a greater susceptibility of the nervous regulation, and a reduction of the performances both in nasal obstruction and in the multifunctionality of the nose.

Nasal mucus felting according to Rolando's classification (1984).

The nasal mucus specimen is obtained atraumatically with a micropipette, without anesthesia, is placed on a slide and left to dry at room temperature $\left(20-22^{\circ} \mathrm{C}\right)$. It's observed under a phase contrast microscope at 40-100 magnifications. The test is based on a characteristic common to all mucous secretions, which is their

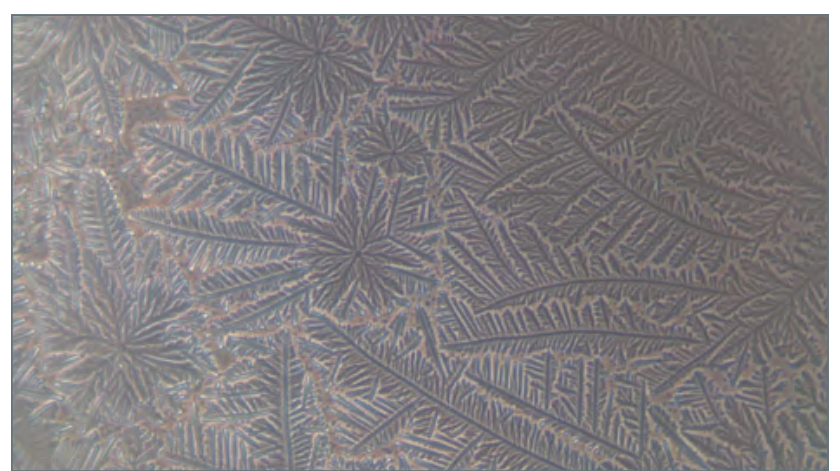

Figure 1. Type I: ferning present uniformly without spaces between the different ones.

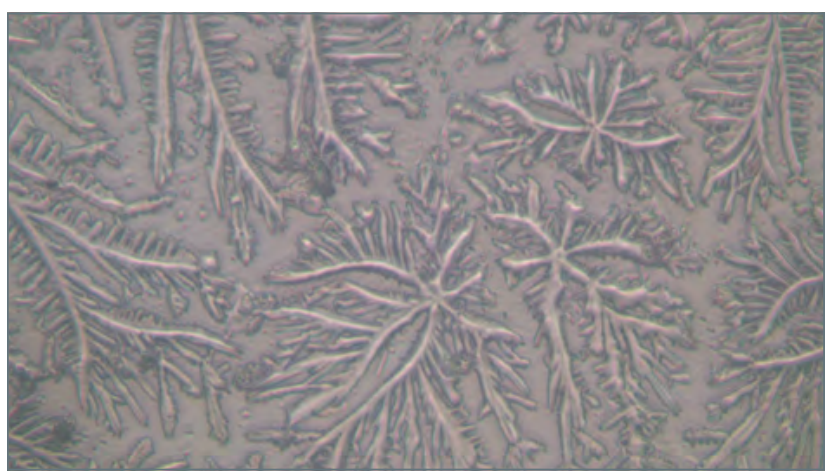

Figure 2. Type II: still abundant crystallization but smaller ferns with small gaps.

ability to crystallize in the form of ferns, when dried, due to evaporation. The biochemical processes of ferning are still not fully known. The most accepted hypothesis proposes that the felting process derives from the interaction between electrolytes $(\mathrm{Na}+\mathrm{Cl}-$ and $\mathrm{K}+$ $\mathrm{Cl}-$ ) with globular material consisting of high molecular weight glycoproteins of mucus (mucins). The optical microscope evaluation defines four types of branching, in particular: Types I and II indicate good mucus and nasal film efficiency. While when the glycoprotein component increases, as it happens during degenerative processes and the consistency of the mucus gets worse, the progress of ferning is prevented (Figs. 3-4).

\section{THE LACRIMAL SYSTEM}

Secretory senescence of the lacrimal system, and its consequence, the dry eye disease, is a very common disorder that affects the elderly population. This causes a defect in the lubrication of the corneal, conjunctival, and scleral mucosa, delicate structures of the external eye, dependent on a failure of the glands of lacrimal system to secrete proteins, lipids and fluid in sufficient quantity or in appropriate composition. The dry eye disease is 
prevalent in women, but this difference becomes relevant only with aging, being senescence the most relevant factor. Asian ethnicity and androgen deficiency are other substantial risk factors for the developing of disease ${ }^{11-12}$. Tear film is essential to maintain the integrity and the health of ocular surface. It is structured in three different layers: the inner layer contains mucin synthesized by conjunctival and corneal cells; the middle layer is composed principally by water, produced primarily by lacrimal glands; the upper layer is composed by lipid secretion released by Meibomian glands. The lipid layer has a physiological key role because it prevents the evaporation of the tear film.

Based on the secretion loss, we can recognize two different types of dry eye disease, linked with the pathogenesis: the first type is the aqueous-deficient, caused by a diminution of the secretion of lacrimal glands, and so the tear output; the second type is linked with the Meibomian gland dysfunction, so it is defined evaporative $^{13}$. Both causes could be represented together: so, tear production decreases and at the same time the composition of the tear film changes.

Different mechanisms related to cellular senescence play a role for the development of secretory loss, causing structural and functional alterations of the lacrimal gland.

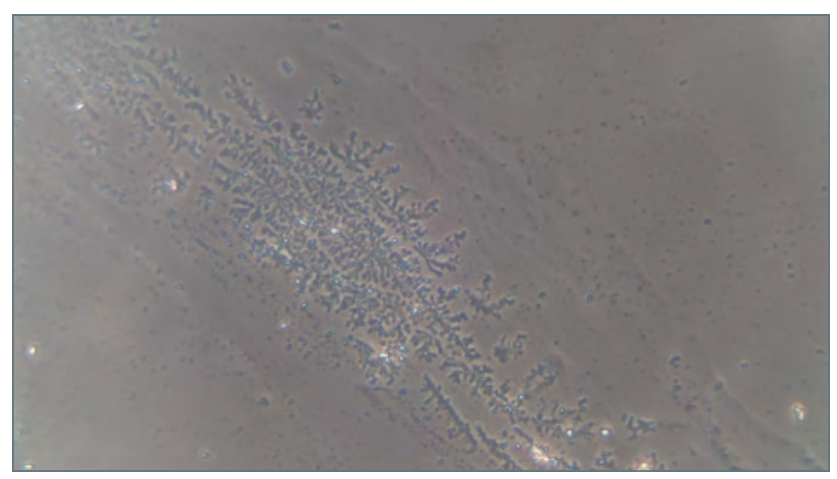

Figure 3. Type III: ferning present only partially.

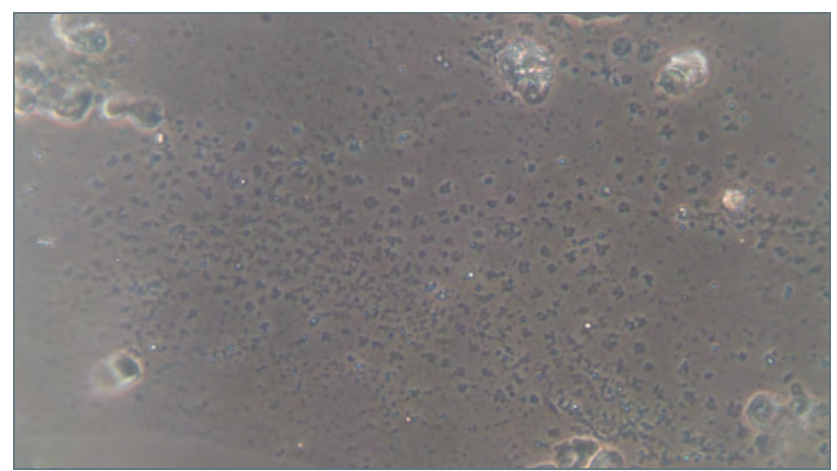

Figure 4. Type IV: dried drop with scattered granules but no ferning.
The oxidative stress produced by free radical agents is one of these principal factors, associated to aging of lacrimal gland cells, altering the structure and function of biological key molecules as lipids, proteins, and DNA. This is evident by intracellular accumulation of lipofuscin, composed by highly oxidized, cross-linked and not degradable proteins ${ }^{14,15}$.

The increase of ROS can also result from hyperglycaemia and cellular resistance to insulin, both conditions frequently encountered in old age people, activating different inflammatory pathway such as NF-kB and JNK/SAPK ${ }^{14-16}$, as demonstrated by Rocha et al. on murine model; furthermore insulin resistance produces reduction in insulin signalling, which include also lipogenesis. In fact, insulin signalling activates an important transcription factor named PPARg, that promotes lipogenesis and sebaceous gland differentiation; this condition could directly affect the activity of Meibomian glands ${ }^{13,14-17}$.

Aging of the immune system and its consequent decrease in efficiency plays another fundamental role, being able to generate a persistent inflammation of the lacrimal pathway, in association with the exposure to numerous external pathogen agents. Thus, chronic and subclinical inflammation created by lymphocytes and polymorphonuclear leukocytes in this anatomical site, for a long period, could generate periductal and perivascular fibrosis, obliteration of blood vessels, and consequentially atrophy of lacrimal glands, associated to the widening and tortuosity of secretory ducts. All these effects have a negative impact on the secretory function ${ }^{18}$.

Some studies demonstrate that minimal alterations start in the middle age and accumulate during life until acinar atrophy and ductal fibrosis produce the initial pathological signs and symptoms ${ }^{4}$.

The function of the Meibomian glands is regulated by the estrogenic, progestin and androgenic hormones, confirming that sequences of mRNA specific for steroid hormones are identified in these structures. Steroid hormones are known to be implicated in the regulation of lipid and protein synthesis, especially androgens are involved in the regulation of sterols, which influence in this tissue the levels of lipid composition and the quantity of secretion ${ }^{19}$. This may explain how low levels of androgen and oestrogen related to aging, can affect the quality and quantity of the tear secretion.

A contribution to the pathogenesis is also given by the nerve fibres. In fact, the epithelium of the ocular mucosa is densely innervated by sensory and visceral nerves, which are finely regulated due to their important contribution to the trophic, protective, and proliferative function, promoting the integrity of the mucosa 19,20 . The senescence process also involves nerve fibres as 
well known, affecting negatively the functionality and homeostasis of lacrimal glands. Peripheral nervous system, in addition, is bidirectionally linked with the immune system as recent studies demonstrate, regulating innate response thought hormonal and neuronal routes. Furthermore, adrenergic nerve fibres work as regulators of the recruitment of leukocytes to and within tissues ${ }^{20,21}$. This issue, combined to what was previously said, could be another additional factor related to the decreased age-related immune response.

The common consequences produced by continue sheer stress on the conjunctival and ocular mucosa are principally discomfort and visual impairment, but in some rare cases corneal perforation that can lead to blindness could occur. Symptoms as dryness, irritation, sensation of foreign body, itching and burning are frequently reported simultaneously. Curiously, signs show a greater increase per decade than symptoms as literature and clinical experience show ${ }^{1}$.

The lacrimal excretory outflow system, through the canaliculus and nasolacrimal duct that ends into lower nasal meatus, also plays an important role in maintaining the health status of this site. Primary or secondary acquired obstruction in this way of output, causes stagnation of secretions with consequential epiphora and superinfection, increasing damage to glandular structures in this site ${ }^{22}$. In these cases, surgical approach is necessary through DCR (dacryocystorhinostomy) to promote a correct drainage of secretions.

Nowadays, several therapeutic approaches are available, due to a better knowledge of the multifactorial mechanisms that lead the disease. Topical therapies with eye drops are certainly the most used to restore the hydration of mucosal epithelium, in association with others topical medications that try to replace deficient biological molecules and reduce inflammation.

First line therapy includes artificial tears with the possible addition of various molecules such as lipids and hyaluronic acid. Cyclosporine is currently used for the treatment of dry eye disease due to its anti-inflammatory properties, reducing IL-2 and T-Cell function, and the capacity of increasing tear production ${ }^{23}$. Another important therapeutic target in dry eye disease are Meibomian glands. Thermal pulsation, warm compress with lipid/semifluorinated alkane-containing eye drops are useful method for this condition; thermal pulsation and intense pulsed light therapy may be suitable as second line therapy in some eligible unresponsive patients ${ }^{24}$. Finally, Lifitegrast is a recently approved class of topical drug. It works blocking the cellular surface proteins LFA-1 (Lymphocyte Function-Associated Antigen 1) and ICAM-1 (Intercellular Adhesion Molecule 1), inhibiting leukocyte migration, and so the symptoms associated to inflammation ${ }^{25}$.

\section{THE TRACHEO-BRONCHIAL TRACT}

Mucus and water are combined to compose the respiratory tract secretions. Vagal efferent nerves regulate the produce of secretions from the airway submucosal glands. This secretion consists of an upper part made of gel and a lower fluid sol in which the cilia beat freely and sweep the gel, with itstrapped inhaled particles, up the airway to be swallowed ${ }^{26}$. Respiratory infections as a major cause of morbidity and mortality in the elderly can be supported by altered and/or insufficient mucociliary clearance, which is impaired in older individuals, but the cause is not completely clear. It depends on the correct functioning of three systems: the synthesis and secretion of mucin and other mucus proteins, the right hydration of the airway surface, and the coordinated activity of the cilia. A perturbation in any of these components, such can occur in the elderly, results in impaired mucociliary clearance ${ }^{27}$.

The elderly people suffer from more frequent and more severe community-acquired and nosocomial infections compared to younger, and this is linked to immunosenescence, physiological aging process, and immune function. In the elderly, the immune system function declines, reducing vaccine responses and increasing susceptibility to infections and to cancer. Thus, immunosenescence is an impairment in both cellular and adaptive immunity as a result of age-related change, causing age-related declines in immune function at both cellular and serologic levels.

Aging is associated with a chronic low-grade inflammatory state and increased proinflammatory cytokines (TNF- $\alpha, \mathrm{IL}-1$, and IL-6).

Furthermore, Toll-Like-Receptors expression and function declines. Toll-Like-Receptors are expressed on a variety of cells not only in blood (including macrophages and lymphocytes) but also in airways (bronchial epithelia) and are utilized by the innate immune system to recognize specific molecular patterns present on pathogenic surfaces. When activated, they can stimulate the secretion of antimicrobial peptides and trigger an inflammatory response through cytokine and chemokine cellular secretion to eliminate the offending pathogen.

There are also significant changes in humoral immune function in the elderly. These changes are characterized by decreased high-affinity antibodies responses and production.

Immunoglobulin $A$ is the dominant immunoglobulin isotype on all mucosal surfaces, including those of the airways, where it acts as a first line of defense against microbial invasion. Secretory lgA concentrations vary over the day due to a range of variables including dietary factors, daily mood, and exercise. Miletic et al. performed a study about IgA secretion in young and old men, and the main finding was that $\lg A$ secretion 
rate was significantly lower in the elderly than in the young ${ }^{28}$.

Finally, a reduction in cell-mediated immunity forms part of the aging immune profile: naïve T-cells are reduced in both the blood and peripheral tissues of elderly individuals, but there is an increase in memory cells, particularly CD4+ and CD8+ ${ }^{29}$.

Cellular senescence is a cell state in which normal cells stop dividing as a mechanism to prevent tumorigenesis and tissue damage. Senescent cells can be cleared by the immune system, but upon aging the number of senescent cells increases in tissues. These cells secrete several inflammatory protein factors and may disturb normal tissue homeostasis and repair. Recent evidence suggests that airway epithelial cells, and particularly Clara cells, play a pro-inflammatory role in the immune response due to secretion of pro-inflammatory cytokines ${ }^{30}$. Clara cell senescence was accompanied by exacerbation of airway inflammation that was at least in part attributable to deregulated pro-inflammatory cytokine secretion by senescent epithelial cells. These findings support the idea that other senescence inducers stimulate pro-inflammatory cytokine secretion.

Pro-inflammatory cytokine secretion is predicted to cause chronic inflammation, at least locally and possibly systemically, becoming one of the complex features of the senescence-associated secretory phenotype, which include alteration of normal tissue histology, promotion of endothelial cell invasion, and stimulation of tumor cell growth and invasion. Airway basal cells represent a wellcharacterized stem cell population located in the trachea and bronchi. These cells have the ability to self-renew and give rise to secretory, ciliated and neuroendocrine cells ${ }^{31}$. Various chronic respiratory disease, first of all chronic bronchitis, have been associated with reduced numbers and dysfunction of circulating progenitor cells. Chronic inflammation and other factors (such as cigarette smoke) have been shown to reduce the repair of endothelial progenitor cells, and so, stem cell exhaustion might contribute to chronic inflammation pathogenesis by reducing the endogenous renewal and repair capacity of the broncho-tracheal epithelium ${ }^{32}$.

\section{References}

1 Ventura MT, Gelardi M, D'Amato A, et al. Clinical and cytologic characteristics of allergic rhinitis in elderly patients. Ann Allergy Asthma Immunol 2012;108:141-4.

2 Preetha $P$, Johnson P, Ramaswamy $P$, et al. The effect of ageing on nasal mucociliary clearance in women: a pilot study. ISRN Pulmonology, vol. 2013.

3 Rolando M. Tear mucus ferning test in normal and keratoconjunctivitis sicca eyes. Chibret Int $\mathrm{J}$ Ophthalmol 1984;2:32-41.
4 Gelardi M, Porro G, Quaranta N, et al. The role of the fern test in the treatment of rhinitis. Rev Alerg Mex 2019;66:18491.

5 Gelardi M, Passalacqua G, Fiorella ML, et al. Nasal cytology: the "infectious spot", an expression of a morphological-chromatic biofilm. Eur J Clin Microbiol Infect Dis 2011;30:1105-9. https://doi.org/10.1007/s10096-011 1198-x

6 Ventura MT, Gelardi M, D'Amato A, et al. Clinical and cytologic characteristics of allergic rhinitis in elderly patients. Ann Allergy Asthma Immunol 2012;108:141-4. https://doi. org/10.1016/j.anai.2012.01.013

7 Mall MA. Role of cilia, mucus, and airway surface liquid in mucociliary dysfunction: lessons from mouse models. J Serosol Med Pulm Drug Deliv 2008;.21:13-24.

8 Houtmeyers SE, Gosselink R, Gayan-Ramirez G, et al. Regulation of mucociliary clearance in health and disease. Eur Respiratory J 1999;13:1177-88.

9 Johnson P, Ramaswamy P, Ramadoss S, et al. The effect of ageing on nasal mucociliary clearance in women: a pilot study. ISRN Pulmonology, Vol. 2013.

10 Kelly FJ, Dunster C, Mudway I. Air pollution and the elderly: oxidant/antioxidant issues worth consideration. Eur Respir J(Suppl) 2003;40:70s-5.

11 Stapleton F, Alves M, Bunya VY, et al. TFOS DEWS II Epidemiology Report. Ocul Surf 2017;15:334-65. https://doi. org/10.1016/j.jtos.2017.05.003

12 El-Fadaly AB, El-Shaarawy EA, Rizk AA, et al. Age-related alterations in the lacrimal gland of adult albino rat: a light and electron microscopic study. Ann Anat 2014;196:33651. https://doi.org/10.1016/j.aanat.2014.06.005

13 Ding J, Sullivan DA. Aging and dry eye disease. Exp Gerontol 2012;47:483-90. https://doi.org/10.1016/j. exger.2012.03.020

14 Rocha EM, Alves M, Rios JD, et al. The aging lacrimal gland: changes in structure and function. Ocul Surf 2008;6:162-74.

15 Tsubota K, Kawashima M, Inaba T, et al. The antiaging approach for the treatment of dry eye. Cornea 2012;31(Suppl 1):S3-8. https://doi.org/10.1097/ ICO.0b013e31826a05a8

16 Evans JL, Goldfine ID, Maddux BA, et al. Oxidative stress and stress-activated signaling pathways: a unifying hypothesis of type 2 diabetes. Endocr Rev 2002;23:599-622.

17 Nien CJ, Massei S, Lin G, et al. Effects of age and dysfunction on human meibomian glands. Arch Ophthalmol 2011;129:462-9. https://doi.org/10.1001/archophthalmol.2011.69

18 de Souza RG, de Paiva CS, Alves MR. Age-related autoimmune changes in lacrimal glands. Immune Netw 2019;19:e3. https://doi.org/10.4110/in.2019.19.e3

19 Lee L, Garrett Q, Flanagan J, et al. Genetic factors and molecular mechanisms in dry eye disease. Ocul Surf 2018;16:206-17. https://doi.org/10.1016/j. jtos.2018.03.003

20 Yamaguchi T. Inflammatory response in dry eye. Invest 
Ophthalmol Vis Sci 2018;59:DES192-DES199. https://doi. org/10.1167/iovs.17-23651

21 Ordovas-Montanes J, Rakoff-Nahoum S, Huang S, et al. The regulation of immunological processes by peripheral neurons in homeostasis and disease. Trends Immunol 2015;36:578-604. https://doi.org/10.1016/j. it.2015.08.007

22 Amin RM, Hussein FA, Idriss HF, et al. Pathological, immunohistochemical and microbiologicalal analysis of lacrimal sac biopsies in patients with chronic dacrocystitis. Int J Ophthalmol 2013;6:817-26. https://doi.org/10.3980/j. issn.2222-3959.2013.06.14

23 Ezuddin NS, Alawa KA, Galor A. Therapeutic strategies to treat dry eye in an aging population. Drugs Aging 2015;32:505-13. https:doi.org/10.1007/s40266-0150277-6

24 Lam PY, Shih KC, Fong PY, et al. A review on evidencebased treatments for Meibomian gland dysfunction. Eye Contact Lens 2020;46:3-16. https://doi.org/10.1097/ ICL.0000000000000680

25 Holland EJ, Darvish M, Nichols KK, et al. Efficacy of topical ophthalmic drugs in the treatment of dry eye disease: a systematic literature review. Ocul Surf 2019;17:412-23. https://doi.org/10.1016/j.jtos.2019.02.012

26 Nadel JA, Davis B, Phipps RJ. Control of mucus secretion and ion transport in airways. Annu Rev Physiol 1979;41:369-81.

27 Grubb BR, Livraghi-Butrico A, Rogers TD, et al. Reduced mucociliary clearance in old mice is associated with a decrease in Muc5b mucin. Am J Physiol Lung Cell Mol Physiol 2016;310:L860-7. https://doi.org/10.1152/ajplung.00015.2016

28 Miletic ID, Schiffman SS, Miletic VD, et al. Salivary IgA secretion rate in young and elderly persons. Physiol Behav 1996;60:243-8.

29 Murray MA, Chotirmall SH. The impact of immunosenescence on pulmonary disease. Mediators Inflamm 2015;2015:692546. https://doi. org/10.1155/2015/692546

30 Regulski MJ. Cellular senescence: what, why, and how. Wounds 2017;29:168-74.

31 Brandsma CA, de Vries M, Costa R, et al. Lung ageing and COPD: is there a role for ageing in abnormal tissue repair? Eur Respir Rev 2017;26. pii:170073. https://doi. org/10.1183/16000617.0073-2017

32 Zhou F, Onizawa S, Nagai A, et al. Epithelial cell senescence impairs repair process and exacerbates inflammation after airway injury. Respir Res 2011;12:78. https://doi. org/10.1186/1465-9921-12-78 